\title{
Computational Explorations and Interpretation of Bonding in Metallo- pharmaceutical for a Thorough Understanding at the Quantum Level
}

\author{
Rajiv Kumar* \\ NIET, National Institute of Medical Science, India \\ E-mail: chemistry_rajiv@hotmail.com
}

Received: 13 March 2021; Accepted: 23 March 2021

\begin{abstract}
Metallopharmaceuticals are effective remedies and used to treat microbial infections. Generally, these drugs were derived from metal-organic frameworks and highly effective to cure many different types of microbial toxicities. In these metallopharmaceuticals, the moieties of the ligands governed novel medicinal properties. Computational explorations (simulations, modeling, and analysis) are the modern tools that explore the possibilities to design novel drugs, analyse the key and essential components required as per the theme of sustainable chemical engineering. To get information at the quantum level, there is an urgent need to explore the associated dynamics that existed in metallopharmaceuticals. These aforementioned strategies will assist to have sustainable chemical engineering models.
\end{abstract}

Keywords: computational explorations, metallopharmaceutical, metal-organic frameworks, effectiveness, experimental investigations, antibiotic characteristics

Nowadays, the techniques of computational simulations and analysis are a key approach to develop sustainable chemical engineering strategies and exploration of associated dynamics. Computational explorations can be engaged to reconnoiter the association between compatibility and innovation. ${ }^{1}$ These discoveries and outcomes can be accomplished via computational simulations to explore the quantum level for developing better practices in sustainable chemical engineering. ${ }^{2}$ Computational simulations, explorations of mechanisms, and ligand-directed selectivities applied for detecting the patterns of bonding in antibiotic drug-metals complexes for a thorough understanding. The self-assemblies of supramolecular architectures display various applications (drug delivery, metallopharmaceuticals, molecular encapsulation, and chemical and photochemical sensing) by developing bioactive assemblies in the fields of drug and medicine. ${ }^{3}$ Computational analysis and apprehensive techniques of explorations have been done to obtain architectural features of metallopharmaceuticals for providing new insights into chemical and biochemical bonding. ${ }^{4}$ These techniques are capable of answering few queries, i.e., how to automate the search for good designs of antibiotic drugs-metals according to the theme of sustainable chemical engineering, how to analyze the consequential sources at the quantum level, and how to make predictions by interpreting the antibiotic drugs-metals bonding patterns for better effectiveness and thorough understanding? A thorough understanding of computational exploration, these techniques is essential for searching the correct answers to the aforementioned queries and a newer sustainable chemical engineering model has been got out of bed. ${ }^{5}$ These discoveries, acquired references and conclusions will prompt the route of the development of novel metallopharmaceuticals according to the requisite models of sustainable chemical engineering (Figure 1). ${ }^{6}$

Copyright (C2021 Rajiv Kumar.

DOI: https://doi.org/10.37256/sce. 212021828

This is an open-access article distributed under a CC BY license

(Creative Commons Attribution 4.0 International License)

https://creativecommons.org/licenses/by/4.0/ 


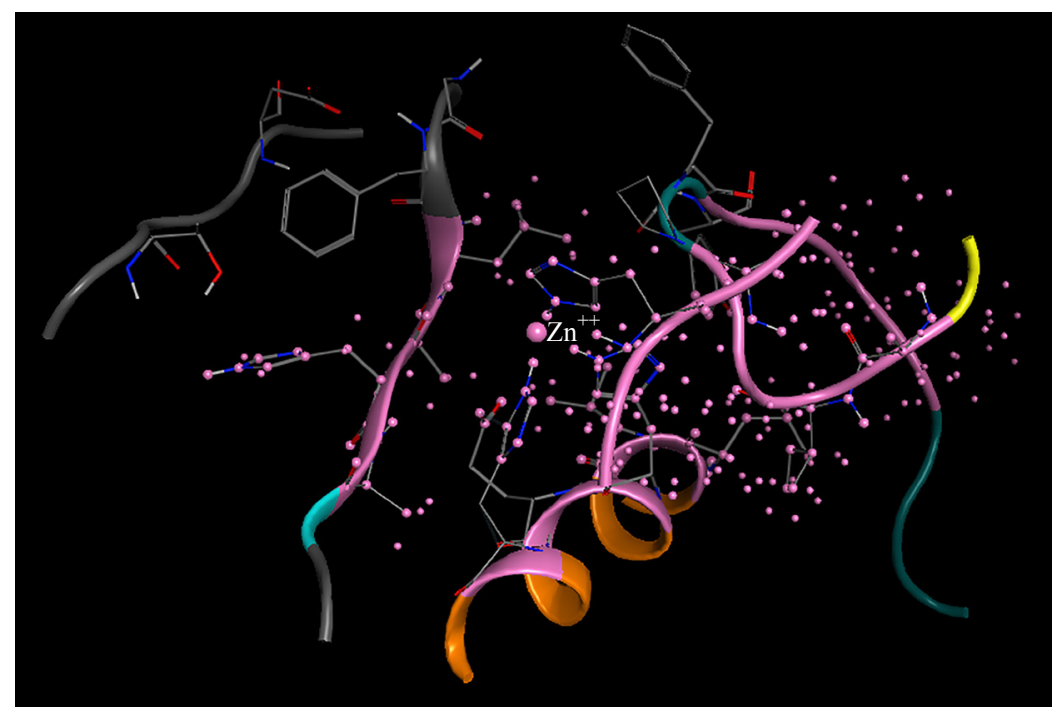

Figure 1. Illustration of architecting and tailoring of computational explorations and interpretation of bonding in metallopharmaceuticals at the quantum level

A better interpretation of bonding will be a key tactic to disclose the effectiveness of such pharmaceuticals at the quantum level. Mechanochemistry deals with the study of metallopharmaceuticals, and bio-inspired metalorganic frameworks which is a smart synthetic technique utilized in metalorganic chemistry for mechanosynthesis of metallopharmaceuticals, explores the medicinal properties that is present on the basis of the existence of metal-drug interactions. These results are applied to uncover the correlation between the electronic structure and reactivity of metallopharmaceuticals derived from metals and antibiotic moieties. The metallopharmaceuticals are becoming highly promising medicine agents because they have a wide variety of features. ${ }^{7}$ The compositions of these derived drugs and medicines (metallopharmaceuticals) have the ability to perform as drug and medicine in a controlled manner in various fields (imaging, diagnostic, therapy), and in other applications.

The well-defined chemistry of metal-ligand particularly is governed by their contact, for example in the antibiotic drugs, where the ligands innovative novel metallopharmaceuticals properties after the formation of various interactions. Therefore, computational exploration and analysis of the formed new bonds between the metal and the donor atoms have been done to interpret the bonding at the quantum level and to explore the potential and quality of the designed metallopharmaceuticals. ${ }^{8}$ These predictions and interpretations will provide a thorough understanding of their existence, stability, effectiveness along with reliable medicinal properties. Such interpretations will define it as a sustainable chemical engineering strategy for better drug development. These advanced analyses were also executed for detecting the binding abilities of a ligand which is depending on the affinities, stability of metal-ligand complexes, electron transfer, and substitution rates. ${ }^{9}$ These tactics are enhancing the possibilities to predict their structures and detecting the biological impact. Computational analysis is a scientific route for noting the specifics of originating perceptions fitted according to the strategy of sustainable chemical engineering to interpret the formed bonds during the formation of metallopharmaceuticals. ${ }^{10}$ A thorough understanding of bonding formation may have the good fortune to obtain useful perceptual evidence simply by noticing what's happening in between, but in many cases, they must assemble and influence things to yield revealing perceptible outcomes. In either case, the observation methodology defines the perceptions or things professed.

Experimental investigations are supporting the theory applied for predicting new metalloantibiotics activity altogether. Several metalloantibiotics drugs were exploited earlier by these methods. Excellent quality of effectiveness was detected. If there is any change in the bonding pattern or removal of the metal ions from the moiety of these antibiotics, it was observed that these alterations change the structural or functional features of these pharmaceuticals at the quantum level. Explorations of the interaction of antibiotic drugs with metal ions existing in metalloantibiotics have been done to determine their uniqueness, effectiveness, and specific bioactivities of these components. ${ }^{11}$ The metalloantibiotics interact with biomolecules and caused various alterations in DNA (Deoxyribonucleic Acid), RNA 
(Ribonucleic Acid), and receptors. Computational exploration of formed new bonds at the time of the formation of the metallopharmaceuticals, and later on when doing alterations, will provide better insight with a thorough understanding at the quantum level. The metallopharmaceuticals and derivatives of metalloantibiotics contain antibiotic characteristics, which displayed microbial-originated metalloantibiotics, antibacterial and antiviral activities, must be investigated at the quantum level to explore the spatial impact of ligands' donor atoms in their architecture. Physicochemical and medicinal properties of referred metallopharmaceuticals are capable of killing the infections of antibiotic-resistant pathogens, ${ }^{12}$ which are posing a non-stoppable threat to humanity.

\section{References}

[1] Kumar, R.; Gulia, K.; Chandra, M.; Aggarwal, A. K.; Kumar, A.; Mittan, S.; Mishra, P. Biomolecular interaction simulation of supramolecular topologies of organometallic assemblies of $\mathrm{Bi}(\mathrm{V})$ with antibiotic Tetracycline Amoxicillin drugs and their experimental activities evaluation. J. Biomed. Ther. Sci. 2019, 6, 61-72.

[2] Kumar, R.; Chauhan, T.; Kumar, A.; Mishra, P. Ecology of supramolecular entities of antibiotics (SMEAs), ondemand controlled guest capture and release systems: tuning spacer and polarities in abiotic and biotic factors. Int. Res. $A d v$. 2017, 4, 61-68.

[3] Kumar, R.; Mishra, P. Spectral analysis, XRPD, quantum structures and evaluation of antimicrobial activity of supramolecular topologies of $\mathrm{Bi}(\mathrm{V})$ and $\mathrm{Co}(\mathrm{II})$ metal based antibiotic drugs complexes. Int. Res. Adv. 2016, 3, 6979 .

[4] Johar, R.; Kumar, R.; Aggarwal, A. K. Tailoring methodologies for the architecture of organometallic frameworks of $\mathrm{Bi}(\mathrm{V})$ derived from antibiotics: Spectral, MS, XRPD and molecular modeling with antifungal effectiveness. $J$. Integr. Sci. Technol. 2013, 1, 54-64.

[5] Gynnild, A. Journalism innovation leads to innovation journalism: The impact of computational exploration on changing mindsets. Journalism 2014, 15, 713-730.

[6] Johar, R.; Kumar, R.; Mishra, P. Molecular modeling of Bi(V)-MCs derived from streptomycin derivatives: synthesis and spectroscopic studies. J. Integr. Sci. Technol. 2015, 3, 18-21.

[7] Hu, Y.; Stumpfe, D.; Bajorath, J. Computational exploration of molecular scaffolds in medicinal chemistry. Journal of Medicinal Chemistry 2016, 59, 4062-4076.

[8] Kumar, R.; Chhikara, B. S. Organometallic assemblies: $\pi$-electron delocalization, $\mu$-bridging spacers, flexibility, lipophilic nature, bio-accessibility, bioavailability, intracellular trafficking pathways and antimicrobial assimilation. J. Organomet. Chem. 2015, 776, 64-76.

[9] Rajiv, K.; Rajni, J. Computational approach on architecture and tailoring of organic metal complexes derived from streptomycin and Zn, Cd and Pb: Antimicrobial effectiveness. Appl. Organomet. Chem. 2011, 25, 791-798.

[10] Hosseinnejad, T.; Ebrahimpour-Malmir, F.; Fattahi, B. Computational investigations of click-derived 1,2,3-triazoles as keystone ligands for complexation with transition metals: A review. RSC Advances 2018, 8, 12232-12259.

[11] Luber, S. Recent progress in computational exploration and design of functional materials. Comput. Mater. Sci. 2019, 161, 127-134.

[12] Laws, M.; Shaaban, A.; Rahman, K. M. Antibiotic resistance breakers: Current approaches and future directions. FEMS Microbiology Reviews 2019, 43, 490-516. 